\title{
Einstieg in den Ausstieg oder Kapitulation vor der Atomwirtschaft?
}

Als die Medien Ende 1992 verbreiteten, daß sich die Stromriesen RWE und VEBA mit dem niedersächischen Ministerpräsidenten Gerhard Schröder (SPD) über die Zukunft der deutschen Atomanlagen verständigt hätten, ging ein Raunen durch die Republik. Sollten die lange geforderte Energiewende und der Ausstieg aus der Atomenergie nunmehr Wirklichkeit werden? Handelt es sich wirklich um den ,Einstieg in den Ausstieg" oder um eine Neuauflage des mißglückten ,historischen“ Atomkompromisses, der 1980 von der Enquete-Kommission „Zukünftige-Kernenergie-Politik“ erwirkt worden war. Der damals verabschiedete detaillierte Katalog zur Senkung der Energienachfrage blieb nahezu folgenlos, während die Atomprojekte zügig vollendet wurden.

Im Sommer 1986, nach der Katastrophe von Tschernobyl, legten sich Sozialdemokratie und Gewerkschaften nach Jahren des Jeins programmatisch auf den Ausstieg fest; seitdem läuft die 10-Jahresfrist. Der von den Grünen geforderte Sofort-Ausstieg, obwohl technisch und wirtschaftlich binnen Jahresfrist machbar, scheiterte bisher an den ",vested interests" der Regierungsparteien. Teilen der SPD war der Ausstiegsbeschluß von Anfang an nicht geheuer, und auch die SPD-geführten. Bundesländer bekleckerten sich bei der Umsetzung bisher keineswegs mit Ruhm.

Im Zeichen der drohenden Klimakatastrophe und angeblichen Vorteilen der Atomkraftwerke beim Treibhausgas $\mathrm{CO}_{2}$ ging die Atomwirtschaft erneut in die Offensive. Von ihr selbst stammt die Forderung nach einem energiepolitischen Grundkonsens. Der zurückgetretene Bundeswirtschaftsminister Möllemann, dessen Ministerium für die bundesdeutsche Energiepolitik hauptverantwortlich ist, griff diese Idee bereitwillig auf. Während das Motiv der Stromkonzerne vor allem darin bestand, keine weiteren Nuklear-Investitionen in den Sand zu setzen, beschwor das Bundeswirtschaftsministerium im Übereinklang mit der Atomlobby die Unverzichtbarkeit der nuklearen Energieversorgung. Im Energieprogramm für das vereinigte Deutschland wird als einzige soziale Innovation die Forderung nach einem ,möglichst parteiübergreifenden Konsens" erhoben und die Einrichtung einer Kommission angekündigt, für die der Bundestag jedoch keine Mittel bereitstellte. Sie sollte zur kooperativen Klärung von Konsensmöglichkeiten beitragen und Optionen für langfristige energiepolitische Strategien erarbeiten.

Den atomaren Hardlinern paßt die ganze Richtung nicht. Bayernwerk, PreussenElektra und die süddeutschen VDEW-Mitglieder halten die Nutzung der Atomkraft für unverzichtbar und dulden keine Gegenmeinung. Sie lehnen den Ausstieg ebenso ab wie eine zeitliche Befri- stung. Sie verstehen unter einem energiepolitischem Konsens schlicht die Kapitulation von SPD und Gewerkschaften in der Atomsache. Da gehen RWE und VEBA-Vorstand schon strategisch zu Werke. Für sie lautet die entscheidende Frage: Wie kann die SPD ohne Gesichtsverlust ihren Nürnberger Atom-Ausstiegsbeschluß revidieren? Wie können die Sozialdemokraten von ihrem Anti-Atom-Programm abrücken, ohne bei ihren Wählern ein für alle Mal unglaubwürdig. zu werden?

Aus den Chefetagen von IG Chemie und IG Bergbau kamen erste Formulierungshilfen. In ihren gemeinsamen Leitsätzen fordern die $\mathrm{Ge}-$ werkschaften den Umstieg auf ,sichere" Atomkraftwerke und daß die Frage der Endlagerung endlich geklärt werde. Ziel der Gewerkschaftsbosse war es, den unseligen Kohle- und AtomPakt zu erneuern und somit die Arbeitsplätze in der Braun- und Steinkohle zu sichern.

Mit diesen guten Ratschlägen im Ohr führte der einstige Anti-Atom-Kämpfer Schröder - noch 1981 begab er sich bei der illegalen Großdemonstration gegen das AKW Brokdorf vor den Bauzaun in den Bereich polizeilicher Maßnahmen - monatelange Gespräche über die nukleare Zukunft Deutschlands mit den Feinden von einst. Sein Hauptmotiv: keine Atomanlagen in Niedersachsen. Aber anstatt eine konsequente Energiesparpolitik einzuleiten und die Rekommunalisierung der Energiewirtschaft in Richtung Stadtwerke zu unterstützen, setzen Schröder und die Stromkonzerne weiterhin auf Angebotserweiterung. In Niedersachsen sollen abgeschaltete Atomkraftwerke der VEBA-Tochter PreussenElektra durch Kohle- oder GasGroßkraftwerke ersetzt werden. Bei Wilhelmshaven soll ein sogenanntes „Verstromungszentrum" für Importkohle gebaut werden. Die von der Klima-Enquete-Kommission geforderte neue Energiepolitik bedeutet dagegen die Entwicklung von Steuerungsinstrumenten für die Nachfrageseite, um insbesondere den Stromverbrauch zu senken.

Der Brief der Strommanager an Bundeskanzler Kohl vom Dezember 92 ist ein Beleg für die energiepolitische Kapitulation des SPD-Spitzenpolitikers. Neben der Forderung nach einem ,überparteilichen Kernenergie-Konsens" behandelt der Deal mit dem SPD-Strategen Schröder folgende Kernpunkte:

- „Ungestörte Restlaufzeiten“ für alle Atomkraftwerke, die in Deutschland in Betrieb sind.

- Offenhalten der Option für den Bau neuer Atomkraftwerke mit verbesserter Sicherheitstechnik.

Verzicht auf die Wiederaufarbeitung abgebrannter Brennelemente und statt dessen direkte Endlagerung.
- Verzicht auf Gorleben als Endlager. Als Alterative kommen Schacht Konrad, Morsleben oder das Ausland in Frage.

- Als Entsorgungsnachweis soll einstweilen die Zwischenlagerung anerkannt werden.

Bewertet man dieses Ergebnis im einzelnen, so beschreibt die Übereinkunft einerseits nur die reale Situation. Seit dem Stopp des Projekts Wackersdorf ist die Wiederaufarbeitung in Deutschland ohnehin gestorben. Eine direkte Endlagerung erfordert jedoch eine Konditionierungsanlage - Kosten mehrere Milliarden Mark -, für die ein Standort in Deutschland kaum zu finden ist. In Gorleben mußte schon Schröders Vorgänger Albrecht erkennen, daß die Atompläne politisch nicht durchsetzbar sind. Angebote des Auslands - d.h. der VR China und der ehemaligen Sowjetunion - für Abnahme der abgebrannten Brennelemente aus deutschen Atomkraftwerken liegen seit Jahren vor. Perestroika, Glasnost und die politischen Veränderungen im Ostblock sind an der dortigen Atomindustrie jedoch spurlos vorübergegangen. Dort besteht nach wie vor ein militärisch-ziviler Atomkomplex, der von Fossilien der BreschnewÄra kontrolliert wird. Wie wirkungsvoll diese Kontrolle ist, läßt sich an den zunehmenden Schiebereien mit nuklearem Material ablesen. Ein Weiterbetrieb der deutschen Atomkraftwerke ist rechtlich aber nur zulässig, wenn der Entsorgungsnachweis vorläufig entfällt.

Nach den nuklearen Fehlinvestitionen Wackersdorf, Kalkar, Hamm-Uentrop und MühlheimKärlich - Gesamtvolumen rund 25 Mrd. DM dürfte in den Konzernetagen der Stromwirtschaft kaum jemand die Traute haben, ein neues Atomprojekt vorzuschlagen, ohne daß dessen Nutzung politisch langfristig garantiert wird. Was liegt näher, als die bestehenden Anlagen möglichst lange weiter $\mathrm{zu}$ betreiben und den Traum von einem inhärent sicheren Reaktor zu beschwören? En passant wird die Regellaufzeit eines Atomkraftwerks von der Stromwirtschaft nun mit 36 Jahren angegeben. Die Wirtschaftlichkeitsberechnungen etwa des TÜV gehen dagegen von einer Laufzeit von 25 bzw. 30 Jahren aus. Die Praxis zeigt: je älter die Atomkraftwerke sind, desto störanfälliger werden sie. Und die Reparaturen werden immer kostspieliger. Stromkunden und Steuerzahler dïrfen die Zeche zahlen, die Gewerkschaften sichern umweltschädliche Arbeitsplätze, die SPD beweist ihre Regierungsfähigkeit. Auf der Strecke bleiben lediglich die Energiewende und der Atomausstieg. An der Atomfront also nicht Neues. Von einer strikten Stromsparpolitik sind wir in der Bundesrepublik noch meilenweit entfernt.

Lutz Mez, Berlin 
(c) 20I0 Authors; licensee IÖW and oekom verlag. This is an article distributed under the terms of the Creative Commons Attribution Non-Commercial No Derivates License (http://creativecommons.org/licenses/by-nc-nd/3.o/), which permits unrestricted use, distribution, and reproduction in any medium, provided the original work is properly cited. 